\title{
https://doi.org/10.48009/2_iis_2007_392-398 \\ STEP CLOSER TO PURE GLOBAL COMPETITION: MASTERING MASTER DATA FOR EMERGING GLOBAL COMPANIES AS THEY IMPLEMENT ERP
}

\author{
G.M. Boff, California University of Pennsylvania, boff@cup.edu \\ M.A. Reese, Robert Morris University, marst24@rmu.edu
}

\begin{abstract}
This paper explores, via literature review and focus group discussions, the relative importance that Master Data Management (MDM) plays in the implementation and maintenance of ERP systems. The focus group included key players of large-scale ERP implementations and/or upgrades, from both an internal intra-company and consulting standpoint. The findings of the discussions resulted in practical advice on developing an efficient and effective MDM process within the ERP system, reducing the risk of a failed implementation or the need for expensive additional data management software. The potential impact on the global marketplace and competition is also explored, as small and medium sized globallypositioned businesses embrace ERP. Given that the challenges of master data are not as great if carefully planned and managed, there is potential for small and medium-sized businesses to gain a competitive advantage over larger competitors.
\end{abstract}

Keywords: ERP, Enterprise Resource Planning, Globalization, Global Competition, Competition, Master Data, Master Data Management.

\section{INTRODUCTION}

The world really is small and becoming smaller as the significance of international borders lessens. Due in no small part to rapid technological advances, globalization most often refers to an "increasing interdependence in the economic, social, technological, cultural, political and ecological spheres” [15]. When considering business-specific components of globalization, such as increased trade and cross-border financial flows and their impact in the operation of national economies, it is virtually impossible to consider globalization without reference to its inherent impact on competition. And likewise, it's almost as difficult to consider competition and strategies in gaining competitive edge in a global marketplace without considering Enterprise Resource Planning (ERP).

Peaking in popularity in the 1990's and still sought after today [3], ERP promises a competitive edge in today's global marketplace through a fully-integrated set of business processes that can offer a real-time pulse on the entity, allowing metrics to be weighed against current market conditions for optimal decision making [4]. The ERP applications market grew from $\$ 25.4$ billion in 2005 to $\$ 29$ billion in 2006, and according to AMR research, is expected to grow an average of $10 \%$ over the next five years [2].

ERP implementations of the past were difficult and expensive, which has, until recently, left small to midsize companies unable to become ERP-enabled. In addition, traditional ERP systems were developed with large-scale manufacturing in mind, which excluded service organizations and specialty manufacturers; in addition, most service organizations are smaller due to the need for subject-area specialization and the lower barrier to entry in the service market than in large-scale manufacturing.

As ERP becomes more attainable to these sorts of companies, the global competitive playing field is evening out, making the potential impact on global competition startling.

One might ask how these smaller companies, who are just undergoing ERP implementations now, could possibly ever catch up with their larger manufacturing forerunners. The answer is by 'doing it better'; and the best way to do it better is to find out where their predecessors tripped up; in other words, to learn from history.

\section{HISTORY OF ERP}

Multi-million dollar, global conglomerates do not emerge unexpectedly on the market - they are grown through acquisition and merger. What results, from an information technology (IT) standpoint, are disparate computer systems, varying business processes and incompatible data: the perfect recipe to create a need for ERP.

As previously mentioned, ERP promises a competitive edge in today's global market through a fullyintegrated, streamlined business [4]. Traditionally, ERP was designed with large, multi-national manufacturing conglomerates in mind. As such, it's 
been this suite of Fortune 500 companies that have taken the ERP plunge and are the current forerunners in the global marketplace.

However, the path to 'true' ERP integration has been riddled with disappointment and millions of dollars toward failed efforts $[5,11]$. With a host of culprits blamed for ERP shortcomings, such as complexity issues [10], cultures resistant to change [13] and the common time and cost constraints [4], AMR Research vice president, Bill Swanton confirms that "half of all problems with ERP systems are caused by master data issues" [1].

Master Data (MD) is today's term for the critical entities of information necessary for organizations to conduct business. MD is comprised of "masters" that represent the harmonized and consistent coding representations for customers, vendors, products and things of the like, and the sub-fields that are related to them. What's more is that these masters and their subfields are not only related to each other, but appear on the hundreds of transactions (sales, inventory, accounting, production planning, etc.) that can occur within ERP when, for example, a sale is made. Data consistency in the masters is what enables the roll-up capability that can extend incredible power to strategic decision makers. The impact of that roll-up capability increases as the globalization of the company increases.

Recent studies in ERP show that the previouslymentioned 'grown' businesses that had taken the ERP plunge and are coming up short are going back to their information-systems roots in an effort to untangle their ERP systems and learn why they cannot rely on their numbers [6]. What they are finding is that the difficulty is not so much in the harmonization of business processes, but more so, in the harmonization of the data that is the foundation to these business processes.

Based on legacy systems, data became, over time, 'intelligent' with tacit meaning embedded in keys and fields that reach beyond the evident. When companies merged to create conglomerates, they did not have to sacrifice their data intelligence because, for the most part, the business units still functioned independently. When the units became integrated through ERP, however, the functionality became harmonized between units that still maintained a strong-hold on their data and its meaning. The results are "data as the master" and not ERP "mastering the data". AMR Research shows that "Among large manufacturing companies, only about $20 \%$ - $25 \%$ have an active strategy for master data [6]. The rest are all wrestling with how to do it." [1].
The price for the lack of attention to MD during ERP implementations [4] as well as the defining of realistic, formalized processes that would need to be put into place to maintain data masters [1] are now being realized and companies, including ERP software vendors such as SAP and Oracle, are responding with “Master Data Management” (MDM). MDM is a paradigm of best practices and/or technological add-on (bolt-on) solutions to ERP systems [7], and is the most popular item of interest in ERP for 2007.

\section{MASTER DATA MANAGEMENT}

"Started in 2003 as a bolt-on module for SAP's NetWeaver, MDM has blossomed into an industry-wide initiative" [12]. MDM software solutions are popping up almost monthly along with professional magazines devoted to the subject and web site portals. The current MDM market size is about $\$ 2$ billion and is expected to grow to $\$ 10$ billion by 2009 according to IDC research data [12]. The top MDM products on the market today are IBM WebSphere Customer Center, Oracle-Siebel Customer Data Hub and Universal Customer Manager, and SAP's NetWeaver MDM.

The premise behind MDM software is for enterprises to have a common view and taxonomy of the still nonstandardized MD that exists throughout their enterprise. In other words, it allows the ERP system to function with the disparate data that it has now by attaching the data with a common tag.

There are three over-arching technical approaches to MDM software with a number of varieties stemming from these three. Termed "hub technologies", these standard MDM approaches are "A Conformed Data Warehouse”, "An Enterprise Repository" and "An Enterprise Registry” [14,16].

In all approaches, there is a centralized and standardized set of attributes that is deemed the 'golden record'; that is, the one set of attributes, common to all functional business areas, for a given MD record, that will always be used to identify that MD record in all forms and variations that it might exist elsewhere.

The Enterprise Registry Model, for example, stores only a key that it generates and the existing keys to associated MD records. Any time changes occur to an MD record, the registry is queried for all other occurrences of the MD object and the changes are proliferated throughout the system. On the other hand is the Enterprise Repository which stores a complete set of overriding MD attributes that serve as the 'single face' of the MD to the user, while the back-end processes continue to function with disparate data. In 
this model, significant changes to MD, either upstream or down, are carefully monitored by a data steward (a data owner responsible for the data integrity). The Conformed Data Warehouse approach is very similar to the Enterprise Repository except that dimensions with overriding MD attributes are built on existing data warehouse records and this is what is used as the 'single face' of MD to the user, with carefully-monitored changes pushed upstream when necessary. The approach could be predetermined by the MDM software that is chosen, and that could be dependent upon the ERP software and the add-on technologies that could be in use.

The significant point here, and a common misconception to MDM newcomers, is that the MDM software does not provide a standardization conversion of the existing MD in your ERP system! These hub technologies equate to an additional layer of functionality that is running on top of your already process-intensive ERP system. The immense complexity of the MDM architecture and survivorship algorithms (logic to determine which updates should occur or which records are the best match) is staggering and it is simply a bandage on a problem that, over time, has become too difficult to fix. But companies running on ERP today may have no choice. With the data so finely weaved into a vast array of system functions that are currently running their businesses, making broadscale MD changes, especially with keys and hierarchies, is both complex and dangerous. It seems reasonable, then, to pay a price in complexity in order to avoid the danger, along with a tangible cost that can reach as much as $\$ 1$ million [12].

And so, back to the question of, "What can we learn from the past to help today's companies seeking ERP enablement 'do it better' so as to catch up with the forerunners in the global market and increase global competition?”. The answer: master the master data, from the start, and avoid the need for MDM software. As one author puts it, "solve the data disparity problem where it should be solved: once, at the source"[14].

\section{METHODOLOGY}

This research began with an extensive literature review to learn what sorts of issues are documented as the major obstacles in large-scale manufacturing conglomerates reaping the benefits of their ERP systems, and ended with all roads leading to MD.

As a follow up to what was learned through the literature, a focus group comprised of 10 individuals, who were key players involved in large-scale ERP implementations and/or upgrades, from both a consulting-firm standpoint as well as from an intracompany, data-management view, was assembled. The focus group technique was chosen since studies on the focus group find that participants learn for one another, and the experience is more stimulating than individual interviews [9].

First, participants were asked general questions concerning the ERP software and the implementation approaches used at their individual institutions, so as to rule out outlying responses that might be due to very unique situations. The majority of questions were devised to focus participant attention to their past and current ERP implementations and any obstacles encountered due to $\mathrm{MD}$, from both an initial conversion/MD creation perspective to a post go-live MD maintenance perspective. In wrap-up to the session, general insights and lessons learned were collected.

The focus group responses were compiled by category (thematized) and compared with the literary findings as a source of validation. The points of interest between these two sources are presented and discussed.

\section{RESULTS}

While the majority of late ERP implementations, at large, do not point to MD as the single reason for delays in their system roll outs, they do indicate that MD was probably one of a number of contributing factors to delays; this was the case with our participants as well, and all participants validated that MD issues crept up post go-live, a few to the extent as causing down-time in their live systems. Of those who responded with specific timeframes that MD contributed to delays in go-live, the average time was approximately 10 months.

With literature surrounding MD proliferating at an exponential rate, it is nearly impossible to determine which is the larger issue documented with MD: the initial composition of MD from legacy data and the conversion/load for go-live, or the post go-live maintenance for the MD. When the participants were asked this question, the majority pointed to the initial MD construction as the larger issue with a smaller subgroup indicating that both really are equally critical. However, when forced to choose between the two issues, they chose the initial MD composition as the more critical issue.

All of the organizations represented by the focus group participants are multi-national in nature. As such, 9 of 10 employ both a global and local configuration and MD organization (one uses strictly a global architecture). For this majority having both global and 
local $\mathrm{MD}$, there were multiple levels of organization that needed to occur in the initial MD creation and standardization, one with MD that would be shared around the world and the other with MD that would be shared throughout an instance (for example, a country). Within the latter, however, were numerous, lateral agreements that needed to be reached to allow all masters to stay synchronized with one another, locally, and with the global master level. As to which challenge was the more difficult, establishing the global or the local MD, more felt that global consensus was more difficult to attain, but almost as many thought local and global consensus to be equally challenging.

When a similar question was asked concerning the ongoing maintenance of $\mathrm{MD}$ and which was more difficult, global or local, the responses were virtually the same; in that, consensus on the MD maintenance process was more difficult to reach globally, with local consensus being almost as challenging.

Whether addressing the creation of MD or the ongoing maintenance, and despite the especially complex infrastructure and synchronization routines that were put in place to handle global and local MD, technical difficulties, in either scenario, were not named as the fundamental difficulty; it was the issue in gaining consensus that was problematic. It would stand to reason, therefore, that when questioned if their ERP software vendor had added any enhancements to address MD, the majority replied that there were none. Again, this is because the problems with MD are typically not technical in nature.

On the other hand, the implementation methodologies used to assist organizations are very much people and process oriented. A collection of planning and tracking mechanisms used by organizations to manage their resources during an ERP implementation, each approach always has some thread of the process focused on MD, as well as change management and education which are people oriented. The breadth and depth of the focus of each thread varies, somewhat, by methodology. So, over time, it would seem reasonable to expect changes in the implementation approaches to assist in the people issues at the core of MD.

The literature is not definitive in determining which implementation methods best handle MD approaches or if any changes had been made to existing approaches that better address MD, overall. Still, participants were asked if the implementation plans that they used had been altered in any way, by either the plan's creator or by their particular organization, in order to better address MD. None reported any known changes to the implementation methodology that they used that would assist with MD. However, most of the participants experienced consecutive ERP implementations, as their companies chose a phased approach, either by subsidiary or by functional module (business area), and as such, they did report changes in their implementation plans, instituted by their organization over time, to better address some MD issues that had arisen in the past. As one participant put it, "I believe that many of the methodologies do not adequately address the change management and cultural impact of change relating to the conversion of the MD. Obtaining agreement on new processes, degrees of standardization to be achieved, and on new organizations necessary to maintain the new data structures is usually not well addressed in the methodologies -- which tend to deal with master data as a technical conversion issue which it is not."

Finally, the group was asked of their familiarity with the term "Master Data Management (MDM)". All gave logical responses to the words "master data management" but none of these pertained to the ERP software add-on solutions being marketed today. What this says is that the fundamental problems organizations encounter with MD, whether it is the creation or ongoing maintenance, cannot be fixed with software. Instead, these MD issues need to be fixed with people and procedures within the implementing organization. If not corrected internally, ERP vendors, consulting firms, and companies developing ERP add-on solutions will continue to gain the dollars which could be used to gain competitive advantage or ROI.

As stated earlier, the MDM software market is about \$2 billion and is expected to grow to $\$ 10$ billion by 2009 [12]. Why? One can only speculate that either the large conglomerates already on an ERP system are unaware of the less than optimal solution that bolt-on software provides, or, these same companies are doing the best they can in a less-than-desirable situation, by putting a bandage on an ERP system that's not giving them reliable data, let alone the competitive advantage for which they'd hoped. Either scenario could have these huge market-share holders heading to a tenuous future market standing.

\section{LEARNING POINTS}

As almost a required exercise, we feel compelled to offer a litany of well-meaning suggestions. The unfortunate reality is that they are not any more earth shattering or innovative than seeing a doctor if you don't feel well. What makes the difference is actually following the good advice of those who have survived the process. 


\section{Start early.}

Form an MD team the moment the project gains approval and begin exploring MD even before an ERP software vendor is selected. As one focus group participant emphatically responded to the question of when to begin considering MD, "From the very beginning!" The best, first step is to identify the sources of MD. This is also a very revealing step as business areas often use, as their masters, data that the IT specialists did not know existed.

\section{Work toward the ideal.}

Determine the producers and consumers of all identified data, and collect and analyze the metadata so that each and every attribute and its composition is known and the meaning of the attribute in the business is understood. When rogue data and processes are uncovered, consider 'staging conversions' by using your existing IT support staff to integrate the processes and data into the mainstream legacy systems. This will eliminate integration steps to ERP later. As focus group participants advised, "The primary data structures and hierarchies should be locked down during the design phase. Data mapping/conversion processing happens well into implementation. For large conversions, mock conversions of data and integration testing will typically improve the quality of the data to be converted."

\section{Set up a governance function.}

This is a two-pronged approach. The first is to appoint data stewards by functional business area. These should be 'owners' of the data who have the knowledge of the current source data and the ability to determine how to transform the source into the MD format. Second, develop a data council of which the data stewards are a part, but which have a well-defined, decision-making process and the ultimate authority to make the hard and necessary decisions surrounding MD in order to keep to deadlines. As stated by a focus group participant, "MD is the foundation on which your ERP is built. This needs to be given consideration both preimplementation and post implementation. The adding and changing of data in the system needs to be closely controlled, for this reason there needs to be a master data control group that is solely responsible for any changes or adds to the master data."

\section{Develop an MD life cycle.}

This should be a joint effort of the data council, the ERP project leads, and upper management from all businesses/subsidiaries involved. Look beyond the initial load and develop a comprehensive change process that includes how MD changes will be authorized and audited. For example, this body may decide to never delete old masters; instead, they may use 'inactivates' to maintain an audit trail and, if necessary, offload to archive after a time threshold. Be sure to build in MD anomaly alert reporting for the stewards and a clear process for each anomaly scenario. And finally, integrate the ability to reverse any activity done to MD.

\section{Test, test and then test.}

As part of the MD life cycle, be sure that system configuration or poor process design is not hindering your MD. Remember, the MD function has to include data origination, data management and data consumption. Applications using/consuming the data have to follow the same standards as a scientific experiment. Transactions must be secure, predictable, and reliable.

\section{Document your documentation!}

Enough cannot be said concerning the criticality of documentation. Starting before Step One, throughout each step, between each step and after the last step, document each process, each decision, and each scenario. In fact, there should be processes and procedures concerning documentation and the need for accompanying documentation at each step in approvals by the governance council. Version control your documentation so that the latest decisions and processes are being followed and hide previous versions so that they are not inadvertently accessed. Consider some type of electronic project notebook that performs this function for you. And remember, if it's not in writing, it never happened.

\section{Enlist others - within careful reason.}

Hiring consultants as a catalyst for the project is a good idea. They can offer methods and tools that can be used for years. The key point is that you use the tools. Once a good example is created, use the template and release the consultants. Don't be afraid to hire clerical help to assist in the documentation process as that costs much less than consultants documenting for you. Consider salaries throughout your organization and align expertise with work that can only be completed by the experts, then use clerical/hired help where practical. 


\section{POTENTIAL IMPLICATIONS ON GLOBAL COMPETITION}

No one can truly predict market reactions to change; at least, not with total accuracy. It is a matter of recognizing similar past patterns in order to forecast future patterns. And in all cases, in all predictions, there are the outliers. So it's important to apply the $80 / 20$ rule when evaluating economic predictions. That is, we will be able to point to $20 \%$ of cases on the market where we KNOW this cannot apply. And we must keep in mind the biggest unknown, which is the human factor. We can never be sure that decision makers will choose the most economically sound decisions since, in reality, many other factors - not the least of which is corporate politics - are in play during the decision-making process.

There are a few key factors which should be considered. First is the fact that ERP implementations of the past were wildly expensive, and as such, only the largest conglomerates were able to make the investment in ERP. Second is the fact that traditional ERP systems were developed with large-scale manufacturing in mind. This left only the largest service organizations willing to spend the time and money to customize ERP systems and become ERP-enabled with their large manufacturing peers. Tied to this fact is the reality that there are more, smaller service organizations than there are smaller manufacturing organizations, by virtue of the service business' need for specialization and the lower barrier to entry in service than manufacturing. Third is the fact that these early ERP implementations were riddled with problems, the residual effects of which are still being felt by the conglomerates that implemented them. These companies are not seeing the promised, competitive benefits of their ERP systems. And finally comes the variable in the economic equation which allows our "what if" analysis; and that is the assumption that the smaller manufacturing and multitude of service organizations that are only now seeking ERP enablement, will be able to 'do it better' through mastering their master data from the start.

The first and obvious evolution is steeper competition in the global service sector. Smaller service companies will now be able to compete with the fewer ERPenabled, large service companies and we could see a gradual shift in market share to the smaller organizations. Increased competition means a drop in prices which smaller companies can absorb better due to lower overall overhead/administrative costs. This could ultimately lead to the breakup of the larger, diversified service organizations, by discipline, so that they will be in a better position to compete with lower prices. A total reorganization of the market players could result as the up-and-coming begin to buy out other newcomers to reestablish the huge market players in the service sector.

But before we see the final stages of market repositioning in the service sector, some changes in manufacturing should be expected as well, that will affect who emerges as the leader in the service sector evolution.

While the barrier to entry in manufacturing is much higher than service, there are small to mid-size manufacturing firms that exist, many of which focus on product specialization. If these companies are able to ERP-enable their businesses more efficiently than their large predecessors, they will be more efficient, business-wise, and see a rise in their bottom line. Recent studies have reached the conclusion that ERP has created "a competitive advantage for SMEs [smalland medium-sized enterprises] by being responsive and agile to change" [8]. The extra revenue could be used to diversify and gradually pull market share from the smaller, specialized lines of the larger manufacturing companies. We could start to see spin-offs in large manufacturing being acquired by newcomers and, again, a market repositioning - this time in the manufacturing sector.

So overall, the decisions that are made by manufacturing concerning their use of services during the repositioning of both the service and manufacturing sectors, and how quickly both are to react to market changes, will impact the evolution of both segments. This is the human factor of which no one can be certain.

While this analysis is in no way comprehensive, it is enough to spark some interesting thoughts on market impact and an increase in global competition, and what that could mean. It can offer some hope to the U.S. market as well. Consider a simple example of the many possible. If manufacturing competition increases, the U.S. may be in a better position to compete in the global market, with a decrease in technical off-shoring. This scenario could positively impact the U.S. job market, and could ultimately affect higher education, as high-school graduates could see a more promising end to the effort of obtaining a college degree.

\section{CONCLUSION}

Dr. W. Edwards Deming, once the quality guru of the U.S., taught us that "you must fix the quality problems at their source or you will be doomed to fix them forever"[14]. Given the excessive time and expense involved in the implementation and maintenance of an ERP solution, it makes great sense to carefully plan the 
master data and its relationship to inherent system processes, both at local and global levels. This provides the opportunity for the ERP implementation to produce a competitive advantage and avert the need for expensive, add-on, commercially available MD solutions at a later date. Rather than taking the Sisyphean approach to MD with a never-ending quality and management battle with your information, work to make it correct from inception.

\section{REFERENCES}

1. Bartholomew, D. (2006). Mastering the Data Monster, Baseline, 63, 55-59.

2. AMR Research, Inc (2006). Research News, October 21, $2006 . \quad$ Available: http://www.tekrati.com/research/News.asp?id7968, Retrieved May 15, 2007.

3. Basu, V. \& Lederer, A.L. (2004). An Agency Theory Model of ERP Implementation. ACM/SIGMIS Conference on Computer Personnel Research, 1, 8-13.

4. Boff, G.M. (2002). Implementing an Enterprise Resource Planning System: What Differentiates the Corporations that Make It from Those that Do Not. UMI Dissertation Publishing, ProQuest Information and Learning.

5. Davenport, T.H. (1998). Putting the Enterprise into the Enterprise System. Harvard Business Review, 76(4), 121-131.

6. Hostetter, S. F., Is Your Data Comparable and Consistent? Financial Executive, 20(8), 58-60.

7. Kobieius, J., Master Data Management: Bringing SOA to the Datacenter. Business Communications Review, 37(2), 50-53.

8. Koh, L. \& Simpson, M. (2005). Change and uncertainty in SME manufacturing environments using ERP. Journal of Manufacturing Technology Management. 16(5/6), 629-653.

9. Morgan, D.L. (1988). Focus groups as qualitative research. Newberry Park, CA: Sage.

10. Nah, F.F., Zuckweiler, K.M., \& Lau, J.L. (2003). ERP Implementation Chief Information Officers' Perceptions of Critical Success Factors. Interaction Journal of Human-Computer Interaction,16, 5-22.

11. Pollock, N., Williams, R., Procter, R. (2003). Fitting Standard Software Packages to Nonstandard Organizations: The 'Biography' of an Enterprise-wide System. Technology Analysis \& Strategic Management, 15,(3), 317-332.

12. Prelmesberger, C. (2007). Change Comes to MDM Space, eWeek, 24(12), 13.

13. Soh, C., Sia, S.K., Tay-Yap, J. (2000). Cultural Fits and Misfits - Is ERP a Universal Solution? Communications of the ACM, 43, 47-51.
14. Thornwaite, W. (2007). Kimball University: Pick the Right Approach to MDM, Intelligent Enterprise, 10(2), 1-6.

15. Wickopedia. (2007). Definition of Globalization. Available: http://en.wikipedia.org/wiki/Globalization, Retrieved May 15,2007.

16. Wolter, R. (2007). Master Data Management (MDM) Hub Architecture, Microsoft Library, Available: $\quad$ http://msdn2.microsoft.com/enus/architecture/bb410798.aspx. Retrieved May 17, 2007. 